\title{
An integrated geometric-graph-theoretic approach to representing origami structures and their corresponding truss frameworks
}

Yao Chen, Ph. D. ${ }^{1}$; Pooya Sareh, Ph. D. ${ }^{2}$; Jiayi Yan ${ }^{3}$; Arash S. Fallah, Ph. D. ${ }^{4}$, Jian Feng, Ph. D. ${ }^{5}$

\begin{abstract}
Origami has provided various interesting applications in science and engineering. Appropriate representations and evaluation on crease patterns play an important role in developing an innovative origami
\end{abstract} structure with desired characteristics. However, this is generally a challenge encountered by scientists and engineers who introduce origami into various fields. As most practical origami structures contain repeated unit cells, graph products provide a suitable choice for the formation of crease patterns. Here, we will employ undirected and directed graph products as a tool for the representation of crease patterns and their corresponding truss frameworks of origami structures. Given that an origami crease pattern can be considered to be a set of directionless crease lines which satisfy the foldability condition, we demonstrate that the pattern can be exactly expressed by a specific graph product of independent graphs. It turns out that this integrated geometric-graph-theoretic method can be effectively implemented in the formation of different crease patterns, and provide suitable numbering of nodes and elements. Furthermore, the presented method is useful in constructing the involved matrices and models of origami structures, and thus enhances configuration processing for geometric, kinematic or mechanical analysis on origami structures.

Keywords: origami pattern; directed graph; origami structures; repetitive fold pattern; pin-jointed structures; graph theory

1 Associate Professor, Key Laboratory of Concrete and Prestressed Concrete Structures of Ministry of Education, and National Prestress Engineering Research Center, Southeast University, Nanjing 211189, China. Email: chenyao@seu.edu.cn

2 Creative Design Engineering Lab, Division of Industrial Design, Faculty of Engineering, University of Liverpool, London Campus, EC2A 1AG, UK. Email: pooya.sareh@livepool.ac.uk

3 School of Civil Engineering, Southeast University, Nanjing 211189, China.

4 Aeronautics Department, Imperial College London, South Kensington Campus, SW7 2AZ, UK.

5 Professor, National Prestress Engineering Research Center, and Key Laboratory of Concrete and Prestressed Concrete Structures of Ministry of Education, Southeast University, Nanjing 211189, China. (corresponding author).Email: fengjian@seu.edu.cn 


\section{Introduction}

Origami is the art of folding 2D materials, such as a flat sheet of paper, into 3D objects with desired shapes.

Since early 1980s, origami has evolved into a fertile scientific field connecting diverse disciplines, creating an enormous variety of new designs with various applications. In recent years, innovative engineering research and mathematical studies have promoted the rapid development of origami as an emerging research field. In particular, the interesting mechanical properties of origami structures have attracted the interest of mathematicians, engineers and scientists [1-5]. For instance, the structural stiffness and geometric shape-shifting properties of some origami structures have attracted considerable attention, be it in fashion, architecture, medicine, or engineering, from airbags to flexible electronics to deployable space structures $[1,6,7]$. Notable theoretical progress has been made in the fields related to origami including tree theory, computational origami theory, optimization methods for rigid origami, and geometric mechanics of origami structures [8].

Recently, using origami techniques, reprogrammable structures have been developed from 2D sheets through folding along well-designed creases. A significant advantage of origami-inspired structures is enhanced flexibility in performance, because their properties are neatly coupled to an alterable crease pattern [3]. Origami-inspired structures with the periodic Miura pattern and the non-periodic Resch pattern have been studied [9]. It has been shown that the Miura fold pattern has a negative in-plane Poisson's ratio. Wei et al. [8] have shown that the in-plane and out-of-plane Poisson's ratios are equal in magnitude, but opposite in sign, independent of material properties. In addition, the strong load bearing capability of the Resch pattern has been demonstrated and attributed to the unique way of folding. Kuribayashi et al. [10] introduced the six-fold origami pattern into biomedical engineering and proposed an origami-inspired stent graft. Hunt et al. [11] studied the buckling mechanism of a thin cylindrical shell under torsion and presented origami patterns with twist buckling. Also, they investigated the critical buckling loads and buckling mechanisms of the equivalent truss models. 
It is worth noting that, either designing a new pattern or investigating specific features of these origami-inspired structures, modeling and involved analysis on the structures are necessary. Thus, configuration processing (or pre-processing) for origami structures is important. Nevertheless, during geometric, kinematic or mechanical analysis, the configuration processing for a large-scale structure is usually tedious and time-consuming. Limited studies have been presented for providing powerful and integrated configuration processing methods for these innovative structures. Kaveh and Koohestani $[12,13]$ have developed graph-theoretical methods for the formation of structural configurations and numerical models. Thereafter, a submodel could be expressed in algebraic forms and different functions could be utilized for the formation of the entire structural model, where the functions mainly contain rotations, translations, reflections and projections, or a combination of these operations. Because many properties of original models can be evaluated by considering those of their submodels (or generators), complex computations can be greatly simplified [14, 15]. As most practical origami structures contain repeated unit cells, graph products provide a suitable choice for the formation of crease patterns and structural models. The degree-4 rigid origami known as the Miura pattern is a classic flat-foldable tessellation which retains a single degree-of-freedom during the folding process. In fact, the secret of the intriguing properties of a folded origami model largely relies on the design of an appropriate crease pattern on the 2D sheet. However, most geometric parameters such as coordinates of the vertices, angles between edge lines and the lengths of edges, which are not important in graph theory, have not been integrated into a conventional graph-theoretic approach. On the contrary, these geometric parameters are very important in the developability, flat-foldability, or rigid-foldability of an origami pattern, and thus they should be somehow incorporated.

Here, an integrated geometric-graph-theoretic framework will be proposed for origami patterns and their corresponding truss frameworks, to include both geometry and connectivity in the mathematical notation. A significance of this work is that we employ some undirected and directed graph products to represent crease 
patterns of origami structures and equivalent pin-jointed structures, to enhance configuration processing for geometric, kinematic or mechanical analysis on origami structures. The weights of nodes and edge lines assigned to the subgraphs are used to describe the geometric configuration of the origami patterns. Appropriate element ordering and nodal numbering, and efficient computations and expressions for involved matrices of the origami structures can be obtained using this geometric-graph-theoretic approach.

The content of this work is as follows. Section 2 describes different types of graph products for representing crease patterns. Then, in Section 3, a series of origami patterns are represented by undirected or directed graph products, to verify the effectiveness of the proposed method. Finally, conclusions are given in Section 4.

\section{Graph Products for the Representation of Crease Patterns}

Given that an origami crease pattern can be considered to be a set of directionless crease lines which satisfy the foldability condition $[16,17]$, here we demonstrate that the pattern can be exactly expressed by a specific graph product of independent graphs. By definition, a graph $\mathrm{S}$ consists of a set of nodes (or vertices) $\mathbf{N}(\mathrm{S})$, and a set of members (or edges) $\mathbf{M}(\mathrm{S})$ [18]. A relation of incidence for the nodes and members of a graph is denoted by an adjacency matrix $\mathbf{A}(\mathrm{S})$. The matrix $\mathbf{A}(\mathrm{S})$ of an undirected graph with $n$ nodes is an $n \times n$ symmetric matrix, whose entry $\mathbf{A}_{i j}(\mathrm{~S})$ in the $i$ th row and $j$ th column is given by

$$
\mathbf{A}_{i j}(\mathrm{~S})= \begin{cases}1 & \text { if node } i \text { is connected to node } j \text { by a member } \\ 0 & \text { otherwise }\end{cases}
$$

A graph is called to be a directed graph, provided that orientations are assigned to its members [14]. Then, a modified adjacency matrix $\overline{\boldsymbol{A}}(\mathrm{S})$ for this directed graph with $n$ nodes can be defined as

$$
\overline{\mathbf{A}_{i j}}(\mathrm{~S})= \begin{cases}1 & \text { if node } i \text { is connected to node } j \text { and directed from node } i \text { to node } j \\ 0 & \text { otherwise }\end{cases}
$$

In Eq. (2), the marix $\overline{\boldsymbol{A}}(\mathrm{S})$ is an $n \times n$ nonsymmetric matrix. 


\section{Cartesian product of undirected graphs}

Many origami structures with degree-4 vertices (e.g., the Miura-ori) have symmetric patterns and thus can be considered to be the Cartesian product of simple graphs $\mathrm{K}$ and $\mathrm{H}$, expressed as $\mathrm{K} \square \mathrm{H}$. In fact, the Cartesian product of subgraphs $\mathrm{K}$ and $\mathrm{H}$ can be formed by taking copies of $\mathrm{H}$ for all the nodes of $\mathrm{K}$ and joining these copies $[13,18,19]$. By using the Boolean operation for the Cartesian product, we can denote the corresponding adjacency matrix $\mathbf{A}(\mathrm{K} \square \mathrm{H})$ as

$$
\mathbf{A}(\mathrm{K} \square \mathrm{H})=\mathbf{A}(\mathrm{K}) \otimes \mathbf{I}_{n_{h}}+\mathbf{I}_{n_{k}} \otimes \mathbf{A}(\mathrm{H})
$$

in which $\mathbf{I}_{n_{k}}$ is the $n_{k} \times n_{k}$ identity matrix, $n_{k}$ is the number of nodes of the graph $\mathrm{K}, \mathbf{I}_{n_{h}}$ is the $n_{h} \times n_{h}$ identity matrix, $n_{h}$ is the number of nodes of the graph $\mathrm{H}$, and $\otimes$ describes the Kronecker product of the matrices. For example, the Cartesian product of two simple graphs $\mathrm{K}=\mathrm{P}_{2}$ and $\mathrm{H}=\mathrm{P}_{3}$ (Fig. 1a-b) is illustrated in Fig. 1(c). Note that a graph $\mathrm{P}$ is known as a path graph whose nodes and members lie on a single straight line [19]. Consequently, the adjacency matrix $\mathbf{A}(\mathrm{K} \square \mathrm{H})$ for the generated graph shown in Fig. 1(c) can be directly computed from Eq. (3), given by

$$
\mathbf{A}(\mathrm{K} \square \mathrm{H})=\left[\begin{array}{ll}
0 & 1 \\
1 & 0
\end{array}\right] \otimes\left[\begin{array}{lll}
1 & 0 & 0 \\
0 & 1 & 0 \\
0 & 0 & 1
\end{array}\right]+\left[\begin{array}{ll}
1 & 0 \\
0 & 1
\end{array}\right] \otimes\left[\begin{array}{lll}
0 & 1 & 0 \\
1 & 0 & 1 \\
0 & 1 & 0
\end{array}\right]=\left[\begin{array}{llllll}
0 & 1 & 0 & 1 & 0 & 0 \\
1 & 0 & 1 & 0 & 1 & 0 \\
0 & 1 & 0 & 0 & 0 & 1 \\
1 & 0 & 0 & 0 & 1 & 0 \\
0 & 1 & 0 & 1 & 0 & 1 \\
0 & 0 & 1 & 0 & 1 & 0
\end{array}\right]
$$

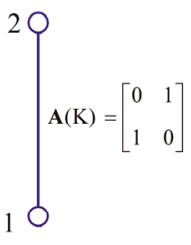

(a) $\mathrm{K}=\mathrm{P}_{2}$

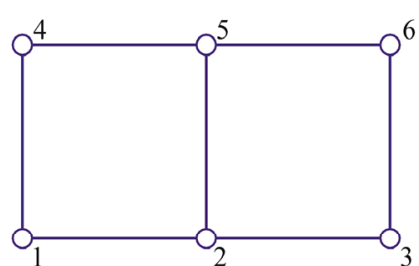

(c) $\mathrm{K} \square \mathrm{H}$

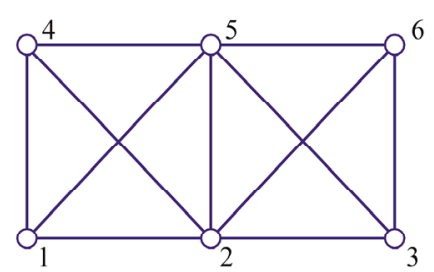

(d) $\mathrm{K} \otimes \mathrm{H}$

Figure 1 The Cartesian product and strong Cartesian product of two simple graphs

\section{Strong Cartesian product of undirected graphs}

The strong Cartesian product of two undirected graphs $\mathrm{K}$ and $\mathrm{H}$ is given by $\mathrm{K} \otimes \mathrm{H}[20]$, which is another type of Boolean operation. The nodes of graph $\mathrm{K}$ are denoted by $i_{k}, j_{k} \in \mathbf{N}(\mathrm{K})$, and a member $i_{k} j_{k} \in \mathbf{M}(\mathrm{K})$ if it is 
connected by two nodes $i_{k}$ and $j_{k}$. Similarly, we denote that a member $i_{h} j_{h} \in \mathbf{M}(\mathrm{H})$ is connected by two nodes $i_{h}, j_{h} \in \mathbf{N}(\mathrm{H})$ of graph $\mathrm{H}$. Then, for different nodes $i=\left(i_{k}, i_{h}\right)$ and $j=\left(j_{k}, j_{h}\right)$ of the strong Cartesian product $\mathrm{K} \otimes \mathrm{H}$, it satisfies

$$
\text { Nodes } i \text { and } j \text { are connected by a member } \begin{cases}\text { if } & i_{k}=j_{k}, \text { and } i_{h} j_{h} \in \mathbf{M}(\mathrm{H}) \\ \text { elseif } \quad i_{h}=j_{h}, \text { and } i_{k} j_{k} \in \mathbf{M}(\mathrm{K}) \\ \text { elseif } \quad i_{k} j_{k} \in \mathbf{M}(\mathrm{K}) \text {, and } i_{h} j_{h} \in \mathbf{M}(\mathrm{H})\end{cases}
$$

In Eq. (5), the nodes $i=\left(i_{k}, i_{h}\right), j=\left(j_{k}, j_{h}\right) \in \mathbf{N}(\mathrm{K}) \times \mathbf{N}(\mathrm{H})$. Thus, the adjacency matrix $\mathbf{A}(\mathrm{K} \otimes \mathrm{H})$ of the strong

Cartesian product is computed by

$$
\mathbf{A}(\mathrm{K} \otimes \mathrm{H})=\mathbf{A}(\mathrm{K}) \otimes \mathbf{I}_{n_{h}}+\mathbf{I}_{n_{k}} \otimes \mathbf{A}(\mathrm{H})+\mathbf{A}(\mathrm{K}) \otimes \mathbf{A}(\mathrm{H})
$$

For example, Fig. 1(d) plots the strong Cartesian product of the above-mentioned graphs $\mathrm{K}=\mathrm{P}_{2}$ and $\mathrm{H}=\mathrm{P}_{3}$. In this figure, the diagonal members exist because the third condition in Eq. (5) is satisfied. In fact, the involved adjacency matrix $\mathbf{A}(\mathrm{K} \otimes \mathrm{H})$ for the graph shown in Fig. 1(d) can be obtained from Eq. (3), written as

$$
\mathbf{A}(\mathrm{K} \otimes \mathrm{H})=\left[\begin{array}{ll}
0 & 1 \\
1 & 0
\end{array}\right] \otimes\left[\begin{array}{lll}
1 & 0 & 0 \\
0 & 1 & 0 \\
0 & 0 & 1
\end{array}\right]+\left[\begin{array}{ll}
1 & 0 \\
0 & 1
\end{array}\right] \otimes\left[\begin{array}{lll}
0 & 1 & 0 \\
1 & 0 & 1 \\
0 & 1 & 0
\end{array}\right]+\left[\begin{array}{ll}
0 & 1 \\
1 & 0
\end{array}\right] \otimes\left[\begin{array}{lll}
0 & 1 & 0 \\
1 & 0 & 1 \\
0 & 1 & 0
\end{array}\right]=\left[\begin{array}{llllll}
0 & 1 & 0 & 1 & 1 & 0 \\
1 & 0 & 1 & 1 & 1 & 1 \\
0 & 1 & 0 & 0 & 1 & 1 \\
1 & 1 & 0 & 0 & 1 & 0 \\
1 & 1 & 1 & 1 & 0 & 1 \\
0 & 1 & 1 & 0 & 1 & 0
\end{array}\right]
$$

\section{Directed graph products}

Type I directed graph product, which is denoted by $(\times)_{1}$ and proposed by Kaveh and Koohestani [13], is utilized to generate triangular patterns for multi-fold origami. For two directed graphs $\mathrm{K}$ and $\mathrm{H}$, any two nodes $i=\left(i_{k}, i_{h}\right)$ and $j=\left(j_{k}, j_{h}\right)$ are connected if one of these six conditions holds: (i) $i_{k}=j_{k}, i_{h} j_{h} \in \mathbf{M}(\mathrm{H})$; $i_{k}=j_{k}, \quad j_{h} i_{h} \in \mathbf{M}(\mathrm{H}) ;(\mathrm{iii}) \quad i_{h}=j_{h}, \quad i_{k} j_{k} \in \mathbf{M}(\mathrm{K}) ;$ (iv) $i_{h}=j_{h}, \quad j_{k} i_{k} \in \mathbf{M}(\mathrm{K}) ;(\mathrm{v}) i_{k} j_{k} \in \mathbf{M}(\mathrm{K}), \quad i_{h} j_{h} \in \mathbf{M}(\mathrm{H})$; (vi) $j_{k} i_{k} \in \mathbf{M}(\mathrm{K}), \quad j_{h} i_{h} \in \mathbf{M}(\mathrm{H})$. It should be noted that, for directed graphs, $i_{k} j_{k} \in \mathbf{M}(\mathrm{K})$ holds if and only if node $i_{k}$ is connected to node $j_{k}$ and directed from node $i_{k}$ to node $j_{k}$.

To effectively generate the directed products of graphs $\mathrm{K}$ and $\mathrm{H}$, we can also concern the corresponding adjacency matrix $\mathbf{A}\left(\mathrm{K}(\times)_{1} \mathrm{H}\right)$, given by 


$$
\mathbf{A}\left(\mathrm{K}(\times)_{1} \mathrm{H}\right)=\mathbf{A}(\mathrm{K}) \otimes \mathbf{I}_{n_{h}}+\mathbf{I}_{n_{k}} \otimes \mathbf{A}(\mathrm{H})+\overline{\mathbf{A}}(\mathrm{K}) \otimes \overline{\mathbf{A}}(\mathrm{H})+\overline{\mathbf{A}}^{\mathrm{T}}(\mathrm{K}) \otimes \overline{\mathbf{A}}^{\mathrm{T}}(\mathrm{H})
$$

where $\mathbf{A}(\mathrm{K})$ and $\mathbf{A}(\mathrm{H})$ denote the adjacency matrices of undirected graphs of $\mathrm{K}$ and $H$, and $\overline{\mathbf{A}}(\mathrm{K})$ and $\overline{\mathbf{A}}(\mathrm{H})$ can be obtained from Eq. (2). Some examples of this product are plotted in Fig. 2, whereas the graphs $\mathrm{S}_{1}$, $S_{2}$ and $S_{3}$ are directed graphs. The member 23 of the directed graphs $S_{2}$ and $S_{3}$ in Fig. 2(b-c) has opposite directions, which indicates the manner for generating the diagonal members.

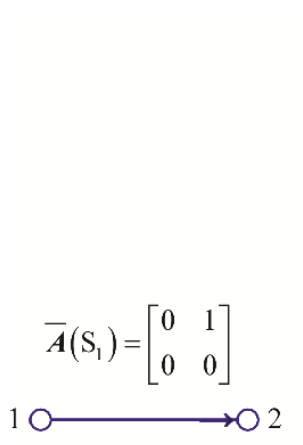

(a) $\mathrm{S}_{1}$

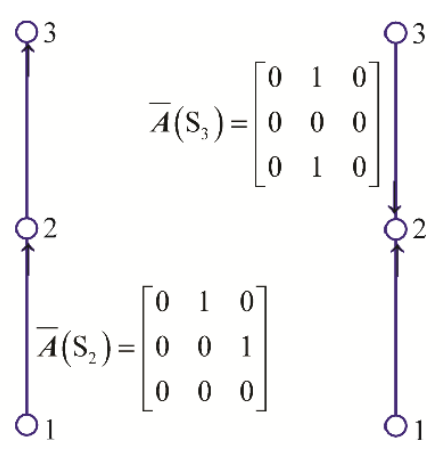

(b) $\mathrm{S}_{2}$

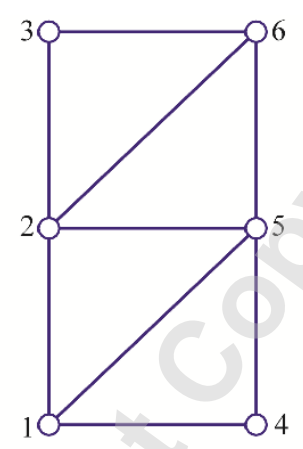

(d) $\mathrm{S}_{1}(\times)_{1} \mathrm{~S}_{2}$

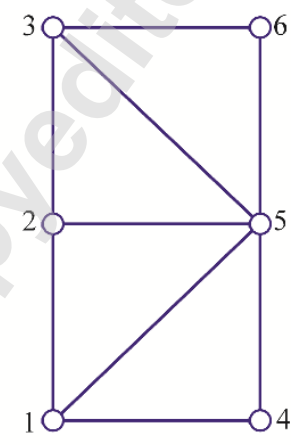

(e) $\mathrm{S}_{1}(\times)_{1} \mathrm{~S}_{3}$

Figure 2 Type I directed graph products of several graphs $\mathrm{S}_{1}, \mathrm{~S}_{2}$ and $\mathrm{S}_{3}$

\section{Algebraic weighted graphs}

Graph products can generate tessellations with new connectivity [13, 15]. However, certain geometric parameters such as angles between edge lines and the lengths of edges, which are not important in graph theory, have not been integrated into a conventional graph-theoretic approach [21-23]. In fact, these parameters are important in the developability, flat-foldability, or rigid-foldability of an origami pattern [24-26], and thus they should be somehow incorporated.

In this case, an integrated geometric-graph-theoretic framework is proposed, to include both geometry and connectivity in the mathematical notation. Weighted graphs, which may be either directed or undirected, are utilized to incorporate the lengths of crease lines. In other words, each edge of a graph is associated with a numerical value, called a weight [22]. Then, an entry $\mathbf{A}_{i j}(\mathrm{~S})$ in the $i$ th row and $j$ th column of a weighted graph $\mathrm{S}$ is defined as follows 


$$
\mathbf{A}_{i j}(\mathrm{~S})= \begin{cases}\eta l_{i j} & \text { if node } i \text { is connected to node } j \text { by a member } \\ 0 & \text { otherwise }\end{cases}
$$

where $l_{i j}$ denotes the length of the involved crease line. To effectively distinguish the directions of the creases for an origami crease pattern, $\eta=1$ in Eq. (9) is denoted for a mountain fold, while $\eta=-1$ for a valley fold. For a non-weighted graph $\eta l_{i j}$ should be replaced by unity. Thereafter, Eq. (9) reduces to either Eq. (1) or Eq. (2). In addition, zero weights should be assigned to the members which need to be removed. In practice, the sum of absolute weights of a graph can be a measure of the complexity of crease processing.

Furthermore, a turning angle between two adjacent edge lines is defined as the weight of the common node, to describe the $2 \mathrm{D}$ geometric configuration of the subgraphs of an origami pattern. That is, the $i$-th entry $\mathbf{N}_{i}(\mathrm{~S})$ of a weighted graph $\mathrm{S}$ in $2 \mathrm{D}$ space is defined as

$$
\mathbf{N}_{i}(\mathrm{~S})=\theta_{i}
$$

where the turning angle $-180^{\circ}<\theta_{i}<180^{\circ}$ is the directed angle (the counterclockwise direction is positive [27]) from vector $\overrightarrow{\mathrm{N}_{i-1} \mathrm{~N}_{i}}$ to vector $\overrightarrow{\mathrm{N}_{i} \mathrm{~N}_{i+1}}$. The notation in Eq. (10) is specifically for path graphs and a general 2D graph, because it can neatly describe the orientation of the graph in 2D space. Note that the turning angles $\theta_{i}$ of the two endpoints of an unclosed graph can be evaluated with respect to $+x$ axis.

In more general cases, nodal coordinates should be associated with a specific node to accurately describe the origami pattern and its subgraphs in 3D space. Then, the $i$-th entry $\mathbf{N}_{i}(\mathrm{~S})$ of a weighted graph $\mathrm{S}$ in 3D space is defined as

$$
\mathbf{N}_{i}(\mathrm{~S})=\left[\begin{array}{lll}
x_{i} & y_{i} & z_{i}
\end{array}\right]^{\mathrm{T}}
$$

where $x_{i}, y_{i}$, and $z_{i}$ denote the coordinates of node $i$ in 3D space.

\section{Further operations employed for graph products}

We have introduced several types of undirected/directed graph products, which are capable of generating a series of origami patterns. Nevertheless, other operations can be further employed to enhance this capability. These 
additional operations include intersection (e.g., $\mathrm{S}_{1} \cap \mathrm{S}_{2}$ ), ring sum (e.g., $\mathrm{S}_{1} \oplus \mathrm{S}_{2}$ ) and union (e.g., $\mathrm{S}_{1} \cup \mathrm{S}_{2}$ ). They are useful for subtracting or adding groups of nodes and/or members. Furthermore, we can define other operations to strengthen these capabilities for the formation of complicated origami patterns. Complementary examples will be presented in the next section.

Note that the operands are assumed to have the same number of nodes. Then, the involved matrices of the resulted graph can be easily computed from the matrices of the operands. Moreover, a node should be deleted, on condition that it is left non-connected to the other nodes [18].

\section{Examples of Origami Structures}

In this section, different types of origami structures are developed using the introduced integrated geometric-graph-theoretic framework.

\section{Crease pattern and truss framework of the Miura-ori}

Figure 3(a) shows the crease pattern of the Miura-ori with $6 \times 6$ basic units. In fact, it can be generated from the Cartesian product of a straight line $\mathrm{P}_{13}$ and a piecewise parallel polylines $\mathrm{G}_{1}$. As the acute angle and edge lengths of the parallelogram for the basic unit in the presented example are respectively $\alpha_{0}=70^{\circ}$ and $l_{h}=l_{v}=l$, the turning angle between the adjacent edge lines of graph $\mathrm{G}_{1}$ is $\theta_{i}= \pm\left(180^{\circ}-2 \alpha_{0}\right)= \pm 40^{\circ}$. All the members of graphs $\mathrm{P}_{13}$ and $\mathrm{G}_{1}$ have the same length, $l$. Moreover, the truss framework corresponding to the Miura-ori, shown in Fig. 3(b), is the strong Cartesian product of graphs $P_{13}$ and $G_{1}$. This origami-based truss structure has 169 nodes and 600 truss members. First-order analysis shows that this equivalent truss structure is both kinematically and statically indeterminate, as the rank of the corresponding $507 \times 600$ equilibrium matrix is 456 . 


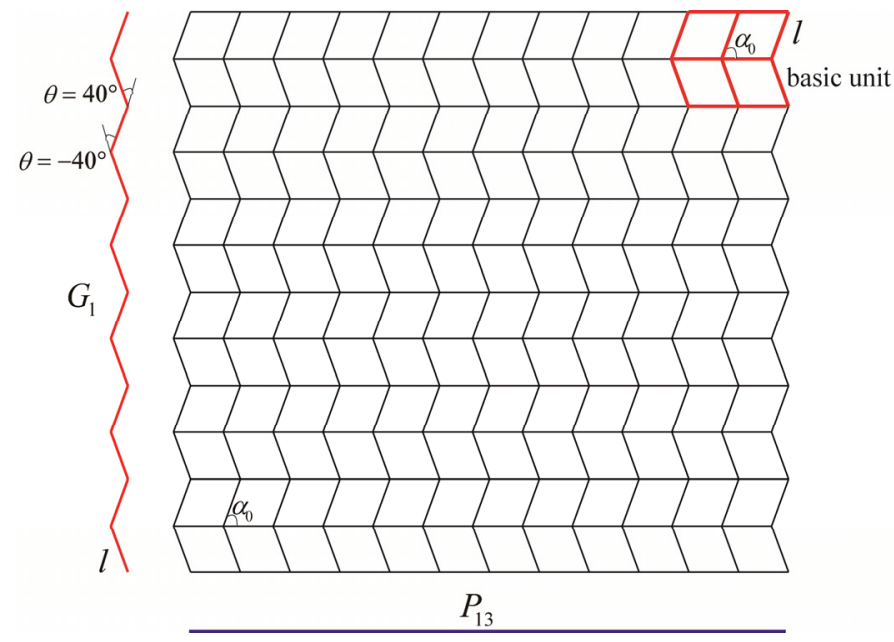

(a) $\mathrm{P}_{13} \square \mathrm{G}_{1}$

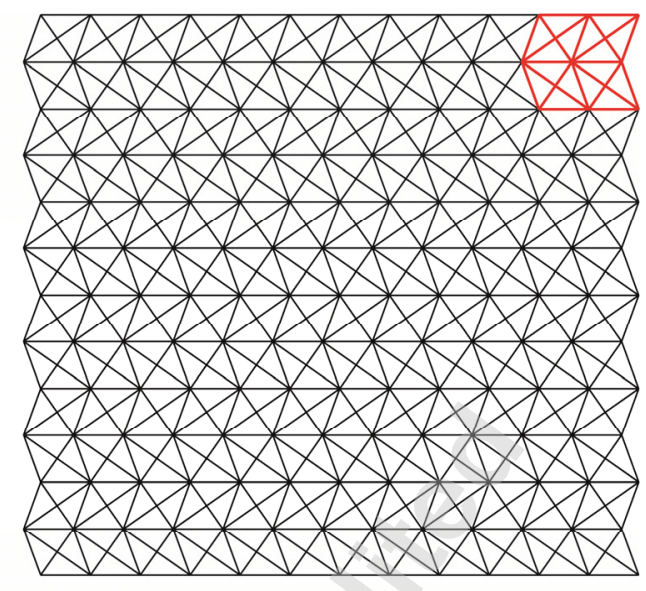

(b) $P_{13} \otimes G_{1}$

Figure 3 Crease pattern and truss framework of the Miura-ori, respectively, denoted by: (a) the Cartesian

product, and (b) the strong Cartesian product of two graphs

Numerical simulation on these origami structures is also performed, to predict the folding motion. Note that the origami model shown in Fig. 3(a) is kinematically investigated using the internal mechanism mode extracted from the equilibrium matrix and the nonlinear prediction-correction method [28, 29]. Besides, the equivalent truss model shown in Fig. 3(b) is analyzed using ABAQUS, whereas each vertex denotes pin-joint node and each member is simulated by a three-node quadratic displacement truss element (to avoid the instability and undesirable deflection of the truss member) [29]. Displacement-based static analysis is utilized for following the folding process, and the geometric nonlinearity is considered. Figure 4 shows typical configurations of the origami model and the corresponding truss framework during transformations. Both structures exhibit similar configurations and transformations during the folding process. Note that the folding is verified to be feasible and approximately rigid-foldable, as the maximum strain of the truss members is less than $1.5 \times 10^{-3}$ along the whole process. This rigid-folding behavior is in good agreement with the results reported by recent studies [17, 29, 30]. Thus, both structural models can simulate the single degree-of-freedom folding behavior of the Miura origami tessellation. Importantly, this Miura-ori structure shows satisfactory folding ratio, whereas the ratio of 
the folded area to the unfolded one is about 0.0451 .
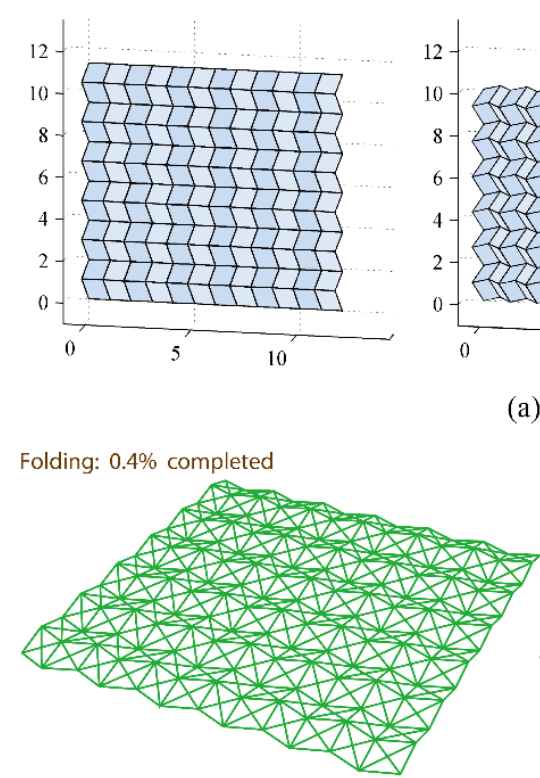

(b) equivalent truss frameworks for predicting the folding behavior Folding: $31.4 \%$ completed Folding: $10.5 \%$ completed
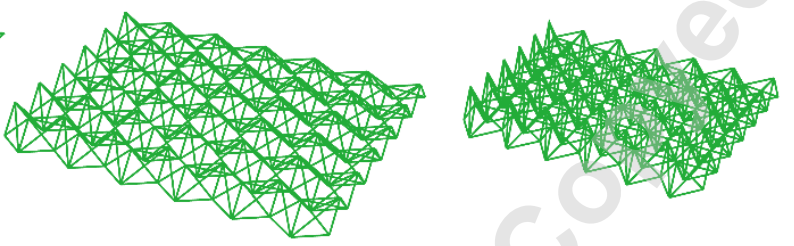
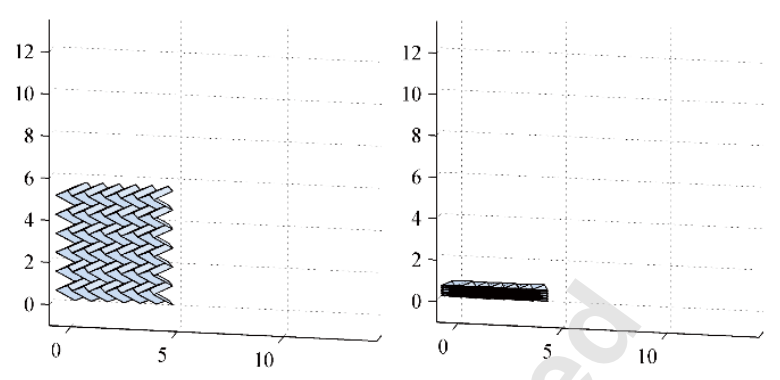

(a) typical structural configurations during folding Folding: $91.7 \%$ completed

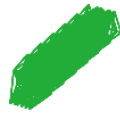

Figure 4 Rigid folding of an origami model with Miura pattern

\section{Representation of different degree-4 origami structures}

The design of appropriate crease patterns is the first step in the development of innovative origami structures with desired characteristics. However, generally this is a challenge encountered by not only origami artists, but also by scientists and engineers who exploit origami patterns in various fields (Tachi [9]; Sareh and Guest [17]). In fact, an integrated mathematical framework for the development of such patterns can be useful as a representational tool, and can inform computer programs generating structural concepts. Here, some degree-4 origami patterns different from the classical Miura-ori are represented by the Cartesian products of different undirected graphs (Fig. 5). 


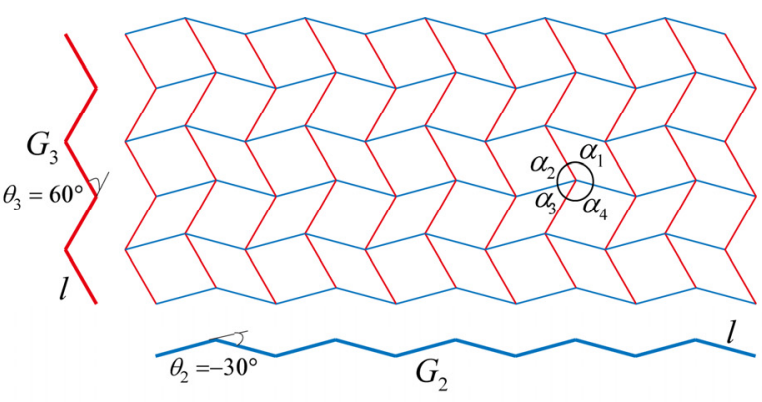

(a) $\mathrm{G}_{2} \square \mathrm{G}_{3}$

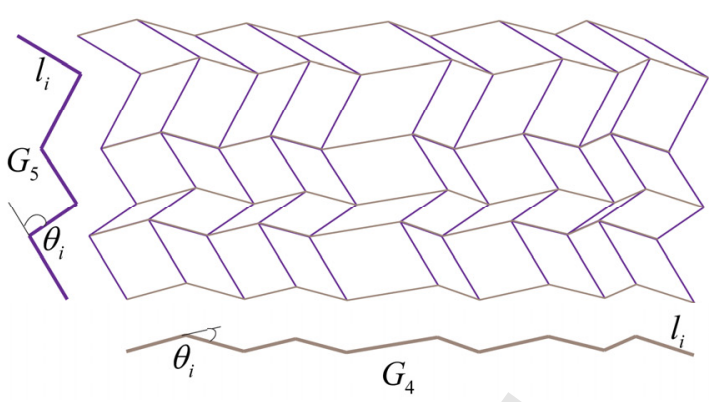

(b) $\mathrm{G}_{4} \square \mathrm{G}_{5}$

Figure 5 Crease patterns of two different degree-4 origami patterns obtained from the Cartesian products of

appropriate subgraphs

The subgraphs $G_{2}$ and $G_{3}$ shown in Fig. 5(a) are the longitudinal and transverse polylines $[16,17,31]$ of the origami tessellations, respectively, where $\theta_{i}\left(G_{2}\right)= \pm 30^{\circ}, \theta_{i}\left(G_{3}\right)= \pm 60^{\circ}$, and the length (weight) of each edge line is $l_{i j}=l$. As the corresponding Cartesian product contains only two types of four-fold vertices, it allows us to determine the flat-foldability in a much easier way. Interestingly, this graph product, which is taken as a modified Miura origami, has been verified to be flat-foldable [25, 31]. As expected, the angles at each four-fold vertex satisfy the following condition

$$
\sum_{i=1}^{4} \alpha_{i}=360^{\circ}, \text { and } \alpha_{1}+\alpha_{3}=\alpha_{2}+\alpha_{4}=180^{\circ}
$$

Eq. (12) can guarantee the local flat-foldability for each vertex of a four-fold origami pattern [25, 31], and it is a necessary condition for the global foldability [32, 33]. For instance, the four angles illustrated in Fig. 5(a) are respectively $\alpha_{1}=135^{\circ}, \alpha_{2}=75^{\circ}, \alpha_{3}=45^{\circ}$, and $\alpha_{4}=105^{\circ}$. Note that the truss structure for this model can be directly obtained from the strong Cartesian product of graphs $\mathrm{G}_{2}$ and $\mathrm{G}_{3}$.

On the other hand, our method can be extended to represent various four-fold origami patterns. Fig. 5(b) shows a more general four-fold origami model than the Miura-ori. It is obtained from the Cartesian product of the graphs $\mathrm{G}_{4}$ and $\mathrm{G}_{5}$, which are two arbitrary polylines. On condition that the rigid-foldability or the flat-foldability of the structure is guaranteed by the reported foldability conditions $[25,31,32]$, the proposed method provides an 
effective way for developing different four-fold origami structures. For example, as far as the local flat-foldability of the origami pattern shown in Fig. 5(b) is concerned, only the angles associated with a small number of vertices need to be evaluated by Eq. (12), because the origami pattern obtained from subgraphs contain periodic cells and limited types of vertices. On the contrary, all vertices should be generally evaluated by conventional methods.

Furthermore, Figure 6 shows distribution patterns of nonzero entries (i.e., nz) of involved matrices for the four-fold origami shown in Fig. 5(a). For instance, the equilibrium matrix $\mathbf{H}$ in Fig. 6(d) is utilized to describe the balance between the internal forces of the members and the external loads [34]. The left zero space of $\mathbf{H}$ contains internal mechanism modes, which can effectively predict the motion of a foldable structure [29, 35]. In Fig. 6, the symbol $\bullet$ is utilized to indicate a non-zero entry.

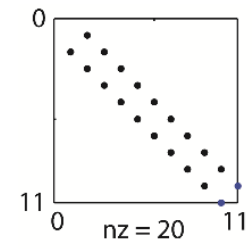

$\mathbf{A}\left(G_{2}\right)$

(a)

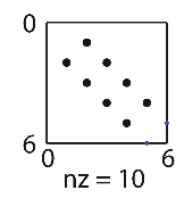

$\mathbf{A}\left(G_{3}\right)$

(b)

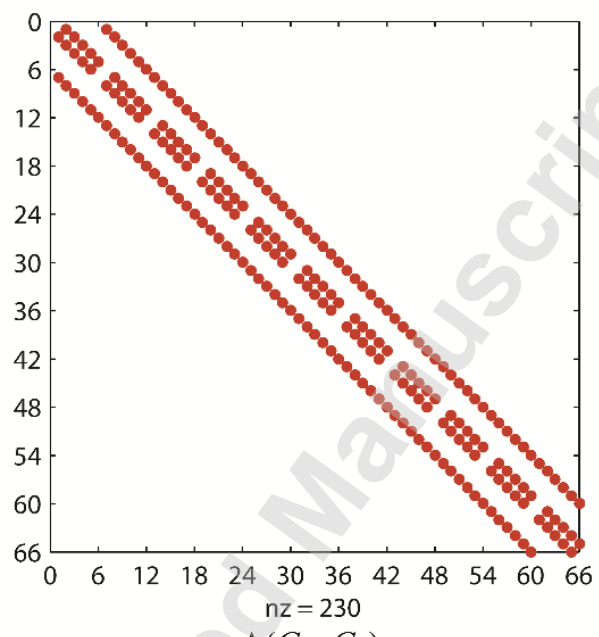

$\mathbf{A}\left(G_{2} \square G_{3}\right)$

(c)

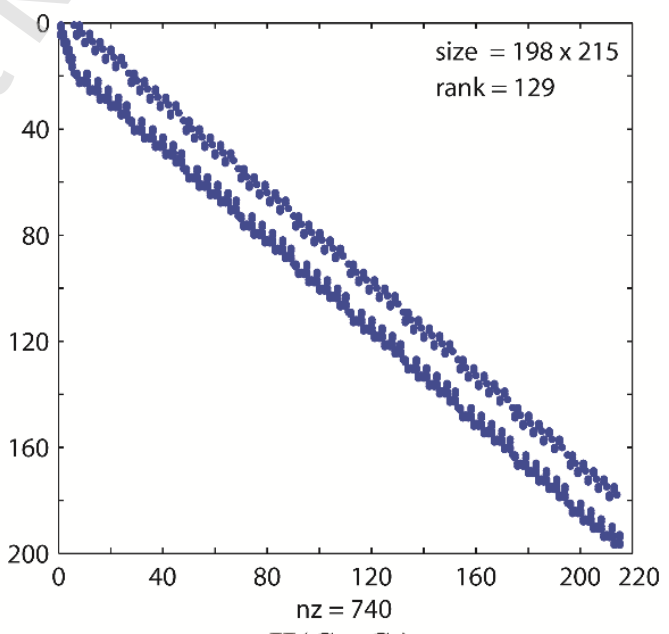

$\mathbf{H}\left(G_{2} \otimes G_{3}\right)$

(d)

Figure 6 Distribution patterns of nonzero entries of involved matrices for the four-fold origami shown in Fig.

5(a): (a-c) adjacency matrices of the subgraphs $\mathrm{G}_{2}, \mathrm{G}_{3}$, and the graph product $\mathrm{G}_{2} \square \mathrm{G}_{3}$, (d) equilibrium matrix of the equivalent truss framework denoted by the strong Cartesian product $\mathrm{G}_{2} \otimes \mathrm{G}_{3}$

It is important to point out that, the involved matrices of the origami models can be evaluated from those of the subgraphs in a much easier manner $[12,15]$. For example, the adjacency matrix shown in Fig. 6(c) exhibits a 
good matrix form and significant regularity. It can be effectively computed from the matrices of the generators of the graph, which are shown in Fig. 6(a-b). In addition, Fig. 6(d) describes the equilibrium matrix of the equivalent truss framework denoted by the strong Cartesian product $\mathrm{G}_{2} \otimes \mathrm{G}_{3}$. Recall that this matrix has a less profile and better distributions of nonzero entries, compared with conventional sparse matrices. This is because graph products for origami structures provide an appropriate element ordering and nodal numbering, which can reduce the profile of involved matrices $[12,13]$. Thus, equilibrium matrices, stiffness matrices and other involved matrices can have better forms and numerical properties.

Kinematic analysis shows that this $198 \times 215$ equilibrium matrix is severely singular. Thus, in the unfolded state, the truss structure of the origami pattern has up to 63 internal mechanism modes, whereas six rigid-body motions have been excluded. As a result, because of potential singularity [28, 36-38], it is difficult to fold the origami structures from the fully deployed state along the ideal motion path.

\section{Six-fold origami patterns based on directed graph products}

As shown in Fig. 7, two different types of six-fold origami models are generated using the directed graph products described in the previous section. Fig. 7(a) plots an origami with Kresling pattern [11], [39]. Interestingly, it comes from the type I product of two directed graphs $D_{\left(1: 8^{+}\right)} P_{9}$ and $D_{\left(1: 8^{+}\right)} G_{9}$, which indicate that the members 1-8 of both directed graphs $\mathrm{P}_{9}$ and $\mathrm{G}_{9}$ with 9 nodes are in positive directions. In this study, the notations $D_{\left(i_{i}, j^{+}, k_{1}: k_{2}^{-}\right)} P_{n}$ and $D_{\left(i_{i}, j^{+}, k_{1}: t k_{2}^{-}\right)} G_{n}$ describe directed path and directed subgraph with $n$ nodes, whereas member $i$, is double, member $j$ is in positive direction and member $k_{1}$ to $k_{2}$ with increment $t$, are in negative direction [13]. 


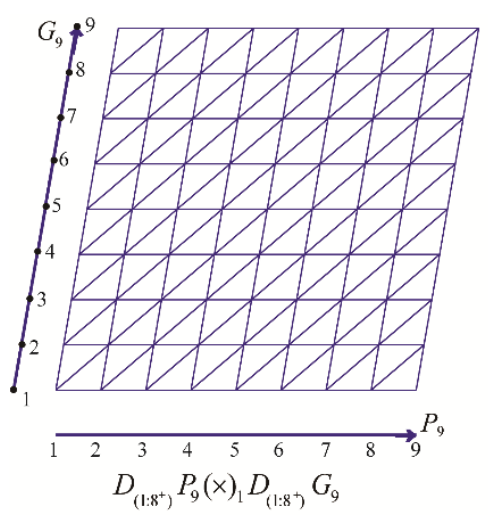

(a) Kresling pattern
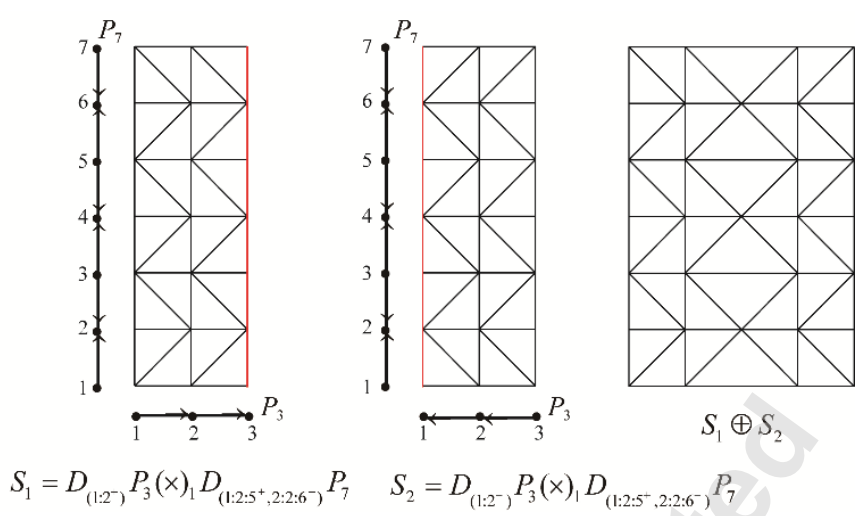

$S_{1} \oplus S_{2}$

$S_{2}=D_{\left.(1: 2)^{-}\right)} P_{3}(\times)_{1} D_{(1: 2: 5,2: 2: 26)^{2}} P_{7}$

Figure 7 Crease patterns of a six-fold origami expressed by directed graph products and further operations

In Fig. 7(b), two directed graph products $S_{1}$ and $S_{2}$ depict two origami patterns with twist buckling modes [11], given by

$$
S_{1}=D_{\left(1: 2^{+}\right)} P_{3}(\times)_{1} D_{\left(1: 2: 5^{+}, 2: 2: 6^{-}\right)} P_{7} \text {, and } S_{2}=D_{\left(1: 2^{-}\right)} P_{3}(\times)_{1} D_{\left(1: 2: 5^{+}, 2: 2: 6^{-}\right)} P_{7}
$$

where $D_{\left(1: 2^{+}\right)} P_{3}$ and $D_{\left(1: 2^{-}\right)} P_{3}$ respectively denote the members 1-2 of the graphs $\mathrm{P}_{3}$ are in positive and negative directions, and the vertical graph $D_{\left(1: 25^{5}, 2: 2: 6^{\prime}\right)} P_{7}$ in Fig. $7(\mathrm{~b})$ denotes that the members 1, 3, and 5 are in positive directions and the members 2, 4, and 6 are in negative directions. As the directions of the horizontal graphs are different, the diagonal members in the generated patterns are different. Moreover, we utilize the operator for the ring sum of the directed graph products $S_{1}$ and $S_{2}$. As a result, a different type of origami model with Waterbomb pattern (Chen et al. [7]; Kuribayashi et al. [22]) is developed. As shown in Fig. 7(b), the intersecting line of the two graphs and the involved nodes have been removed.

Notably, by combining with cycles, weighted graphs and subgraphs, we can develop crease patterns for 3D origami structures with complex geometry. As shown in Fig. 8, crease patterns of cylinders associated with the Kresling pattern are studied. 


$$
\overline{\mathbf{A}}\left(C_{6}\right)=\left[\begin{array}{llllll}
0 & 1 & 0 & 0 & 0 & 0 \\
0 & 0 & 1 & 0 & 0 & 0 \\
0 & 0 & 0 & 1 & 0 & 0 \\
0 & 0 & 0 & 0 & 1 & 0 \\
0 & 0 & 0 & 0 & 0 & 1 \\
1 & 0 & 0 & 0 & 0 & 0
\end{array}\right]
$$

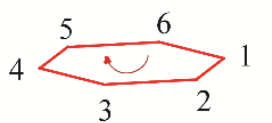

(a)

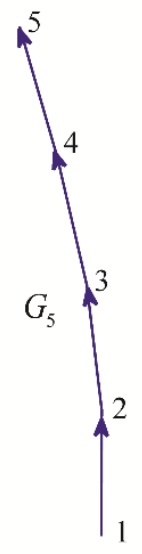

(b)

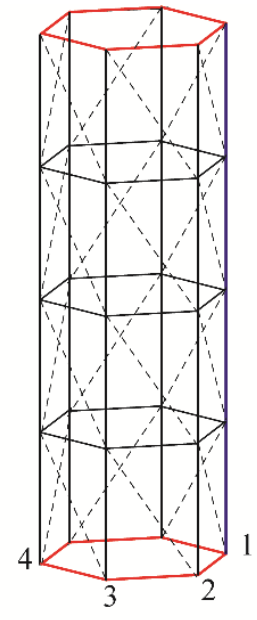

(c)

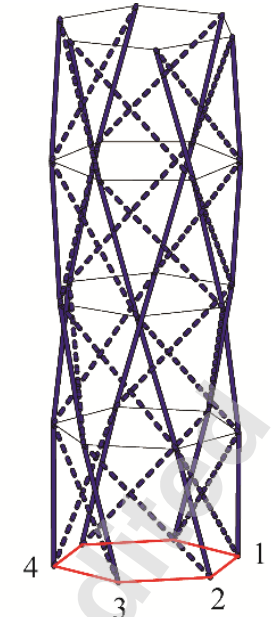

(d)

Figure 8 Cylinders expressed by directed graph products of weighted graphs in 3D space: (a) a directed cycle $D_{1: 6^{6}} C_{6}$ and its modified adjacency matrix $\bar{A}\left(\mathrm{C}_{6}\right) ;$ (b) a weighted graph $D_{1: 4^{+}} C_{5}$ in 3D space; (c) a typical cylinder obtained by $D_{1: 6^{+}} C_{6}(\times)_{1} D_{1: 4^{+}} P_{5}$; (d) a twisted cylinder with the Kresling pattern generated by

$$
D_{1: 6^{+}} C_{6}(\times)_{1} D_{1: 4^{+}} G_{5}
$$

Conventional cylinders such as the one shown in Fig. 8(c) can be directly generated from the directed cycle and path graphs, given by

$$
S=D_{1: 6^{6}} C_{6}(\times)_{1} D_{1: 4^{+}} P_{5}
$$

where the geometry of the graphs is not integrated. In fact, as shown in Fig. 8(d), a specific cylinder with the Kresling pattern can be obtained by twisting a conventional thin cylinder [11, 39, 40]. It retains cyclic symmetry $[41,42]$, and keeps equivalent under proper rotations along the principal axis. Its crease pattern can be accurately established by

$$
S=D_{1: 6^{6}} C_{6}(\times)_{1} D_{1: 4^{+}} G_{5}
$$

where the subgraph $G_{5}$ is a directed weight graph in 3D space. Based on Eq. (11), the coordinates of each node of the graph are wriiten as

$$
\mathbf{N}_{i}\left(G_{5}\right)=\left[\begin{array}{lll}
x_{i} & y_{i} & z_{i}
\end{array}\right]^{\mathrm{T}}=\left[\begin{array}{lll}
r \cos \left(i \theta_{t}-i\right) & r \sin \left(i \theta_{t}-i\right) & (i-1) h
\end{array}\right]^{\mathrm{T}}, \quad i \in[1,5]
$$


where $r$ is the radius of the cylinder, $\theta_{t}$ is the twisted angle between the layers of the cylinder, $h$ is the height of one layer, and they satisfy [40]

$$
h=r \theta_{t} \tan (\delta)
$$

In Eq. (17), $\delta$ is the angle between the weighted graph shown in Fig. 8(b) and the horizontal plane, and indicates the twisted state of the cylinder with the Kresling pattern [40]. For instance, $\delta=0$ when the cylinder is folded. It is important to point out that Eq. (17) is based on infinitesimal bar stretching assumption (i.e., undeformed bar length is utilized to describe the global geometry of the structure).

Based on the graph product computed by Eq. (15) and the FEM analysis using ABAQUS, the equivalent truss framework of the twisted cylinder with the Kresling pattern (see Fig. 8d) is investigated to study the deformation process. Figure 9 illustrates four typical configurations of the cylinder in the deformation process, where $r=1000 \mathrm{~mm}$ and $h=1200 \mathrm{~mm}$ at the initial configuration. It shows that this cylinder can be fully folded from the initial configuration, where the bar members get stresses when deformed. Notably, the deformation process agrees well with the results presented by Cai et al. [40]. Therefore, the proposed graph products provide a good choice for numerical models or finite element modeling of the origami structures.

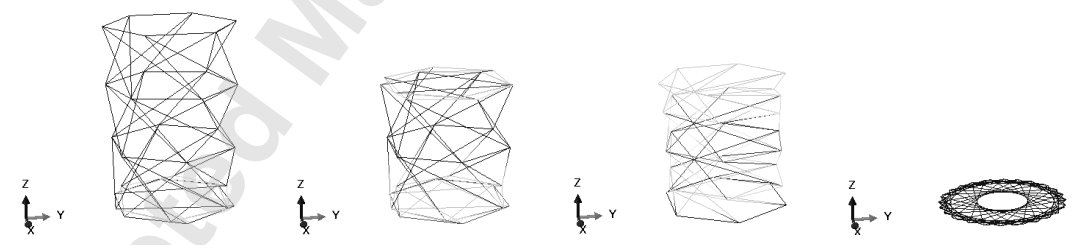

Figure 9 Deformation process of truss framework of a cylinder with the Kresling pattern

\section{Conclusions and Discussions}

Using undirected and directed graph products, we can describe a large variety of origami patterns (e.g., the Miura pattern, different four-fold patterns, Kresling pattern, and Waterbomb pattern), and easily construct the involved matrices and simplified structural models of these structures. On the basis of the graph products and the properties of their generators, the topological properties of origami models can be evaluated in a much easier 
manner. For example, the adjacency matrices of the models can be computed from those of their generators.

Furthermore, the presented method can be extended to the formation of other types of structures and their numerical models. Using different operators of graph products, we can obtain the compact representation of origami structures with complex patterns. More importantly, because the graph products and their operators introduced in this study are systematic, computer programs would be easily generated and exploited. Admittedly, the origami patterns described by the present approach are somewhat periodic. Robust methods for more general patterns with complex geometries or non-periodic characters should be concerned in the future study.

\section{Acknowledgements}

Work supported by the National Natural Science Foundation of China (Grant No. 51850410513 and No. 51508089), and the Fundamental Research Funds for the Central Universities. The first author would like to acknowledge financial support from the Alexander von Humboldt-Foundation for his visiting research at Max-Planck-Institut für Eisenforschung GmbH, Germany. The authors are grateful to the anonymous reviewers for their valuable comments.

\section{References}

[1] Yasuda, H., and Yang, J., 2015, "Reentrant origami-based metamaterials with negative poisson's ratio and bistability," Physical Review Letters, 114(18), 185502.

[2] Brunck, V., Lechenault, F., Reid, A., and Adda-Bedia, M., 2016, "Elastic theory of origami-based metamaterials," Physical Review E, 93(3), 033005.

[3] Silverberg, J. L., Evans, A. A., McLeod, L., Hayward, R. C., Hull, T., Santangelo, C. D., and Cohen, I., 2014, "Using origami design principles to fold reprogrammable mechanical metamaterials," Science, 
345(6197), pp. 647-650.

[4] You, Z., 2014, "Materials design. Folding structures out of flat materials," Science, 345(6197), pp. 623-624.

[5] Chen, Y., Peng, R., and You, Z., 2015, "Origami of thick panels," Science, 349(6246), pp. 396-400.

[6] Chen, Y., Feng, H. J., Ma, J. Y., Peng, R., and You, Z., 2016, "Symmetric waterbomb origami," Proceedings of the Royal Society A-Mathematical Physical and Engineering Sciences, 472(2190), 20150846.

[7] Zhang, X., Cheng, G., You, Z., and Zhang, H., 2007, "Energy absorption of axially compressed thin-walled square tubes with patterns," Thin-Walled Structures, 45(9), pp. 737-746.

[8] Wei, Z. Y., Guo, Z. V., Dudte, L., Liang, H. Y., and Mahadevan, L., 2013, "Geometric mechanics of periodic pleated origami," Physical Review Letters, 110(21), pp. 325-329.

[9] Tachi, T., 2009, "Generalization of rigid foldable quadrilateral mesh origami," Journal of the International Association for Shell \& Spatial Structures, 50(162), pp. 173-179.

[10] Kuribayashi, K., Tsuchiya, K., You, Z., Tomus, D., Umemoto, M., Ito, T., and Sasaki, M., 2006, "Self-deployable origami stent grafts as a biomedical application of Ni-rich TiNi shape memory alloy foil," Materials Science and Engineering A-Structural Materials Properties Microstructure and Processing, 419(1-2), pp. 131-137.

[11] Hunt, G. W., and Ario, L., 2005, "Twist buckling and the foldable cylinder: an exercise in origami," International Journal of Non-Linear Mechanics, 40(6), pp. 833-843.

[12] Kaveh, A., 2013, Optimal analysis of structures by concepts of symmetry and regularity, Springer.

[13] Kaveh, A., and Koohestani, K., 2008, "Graph products for configuration processing of space structures," Computers \& Structures, 86(11), pp. 1219-1231.

[14] Kaveh, A., and Rahami, H., 2005, "A unified method for eigendecomposition of graph products," 
Communications in numerical methods in engineering, 21(7), pp. 377-388.

[15] Chen, Y., and Feng, J., 2016, "Improved symmetry method for the mobility of regular structures using graph products," Journal of Structural Engineering, 142(9), 04016051.

[16] Sareh, P., and Guest, S. D., 2015, "A Framework for the symmetric generalisation of the Miura-ori," International Journal of Space Structures, 30(2), pp. 141-152.

[17] Sareh, P., and Guest, S. D., 2015, "Design of isomorphic symmetric descendants of the Miura-ori," Smart Materials and Structures, 24(8), 085001.

[18] Kaveh, A., and Nikbakht, M., 2010, "Improved group-theoretical method for eigenvalue problems of special symmetric structures, using graph theory," Advances in Engineering Software, 41(1), pp. 22-31.

[19] Kaveh, A., and Alinejad, B., 2009, "A general theorem for adjacency matrices of graph products and application in graph partitioning for parallel computing," Finite Elements in Analysis and Design, 45(3), pp. 149-155.

[20] Sabidussi, G., 1959, "Graph multiplication," Mathematische Zeitschrift, 72(1), pp. 446-457.

[21] Koohestani, K., 2012, "Exploitation of symmetry in graphs with applications to finite and boundary elements analysis," International Journal for Numerical Methods in Engineering, 90(2), pp. 152-176.

[22] Fan, R. K. C., 1997, Spectral graph theory, American Mathematical Society.

[23] Sareh, P., and Guest, S. D., 2014, "Designing symmetric derivatives of the Miura-ori," Advances in Architectural Geometry, pp. 233-241.

[24] Gattas, J. M., Wu, W., and You, Z., 2013, "Miura-base rigid origami: parameterizations of first-level derivative and piecewise geometries," Journal of Mechanical Design-Transactions of the ASME, 135(11), 0111011.

[25] Tachi, T. 2010. "Geometric considerations for the design of rigid origami structures," Proceedings of the 
International Association for Shell and Spatial Structures (IASS) Symposium, pp. 458-460.

[26] Sareh, P., and Guest, S. D., 2015, "Design of non-isomorphic symmetric descendants of the Miura-ori," Smart Materials and Structures, 24(8), 085002.

[27] Ackerman, E., Fulek, R., and Th, C. D., 2012, "Graphs that admit polyline drawings with few crossing angles," SIAM Journal on Discrete Mathematics, 26(1), pp. 305-320.

[28] Chen, Y., Feng, J., and Ren, Z., 2016, "Numerical approach for detecting bifurcation points of the compatibility paths of symmetric deployable structures," Mechanics Research Communications, 71, pp. 7-15.

[29] Chen, Y., and Feng, J., 2012, "Folding of a type of deployable origami structures," International Journal of Structural Stability and Dynamics, 12(6), 1250054.

[30] Filipov, E. T., Liu, K., Tachi, T., Schenk, M., and Paulino, G. H., 2017, "Bar and hinge models for scalable analysis of origami," International Journal of Solids and Structures, 124, pp. 26-45.

[31] Sareh, P. 2014. Symmetric descendants of the Miura-ori. University of Cambridge, UK.

[32] Demaine, E. D., and O'Rourke, J., 2008, Geometric folding algorithms: linkages, origami, polyhedra, Cambridge University Press.

[33] Akitaya, H., D. Demaine, E., Horiyama, T., Hull, T., Ku, J., and Tachi, T., 2018, "Rigid Foldability is NP-Hard," arXiv:1812.01160.

[34] Chen, Y., Feng, J., Ma, R., and Zhang, Y., 2015, "Efficient symmetry method for calculating integral prestress modes of statically indeterminate cable-strut structures," Journal of Structural Engineering, 141(10), 04014240 .

[35] Chen, Y., Fan, L., and Feng, J., 2017, "Kinematic of symmetric deployable scissor-hinge structures with integral mechanism mode," Computers \& Structures, 191, 140-152. 
[36] Chen, Y., Feng, J., and Sun, Q., 2018, "Lower-order symmetric mechanism modes and bifurcation behavior of deployable bar structures with cyclic symmetry," International Journal of Solids and Structures, 139-140, pp. 1-14.

[37] Silverberg, J. L., Na, J. H., Evans, A. A., Liu, B., Hull, T. C., Santangelo, C. D., Lang, R. J., Hayward, R. C., and Cohen, I., 2015, "Origami structures with a critical transition to bistability arising from hidden degrees of freedom," Nature Materials, 14(4), pp. 389-393.

[38] Waitukaitis, S., Menaut, R., Chen, B., and van Hecke, M., 2015, "Origami multistability: from single vertices to metasheets," Physical Review Letters, 114(5), 055503.

[39] Kresling, B., 2008, "Natural twist buckling in shells: from the Hawkmoth's bellows to the deployable kresling-pattern and cylindrical Miura-Ori"., 6th International Conference on Computation of Shell \& Spatial Structures (IASS-IACM 2008), Ithaca, NY, May 28-31.

[40] Cai, J., Deng, X., Zhang, Y., Feng, J., and Zhou, Y., 2016, "Folding behavior of a foldable prismatic mast With Kresling origami pattern," Journal of Mechanisms \& Robotics-Transactions of the ASME, 8, 031004.

[41] Chen, Y., and Feng, J., 2012, "Generalized eigenvalue analysis of symmetric prestressed structures using group theory," Journal of Computing in Civil Engineering, 26(4), pp. 488-497.

[42] Chen, Y., Sareh, P., Feng, J., and Sun, Q., 2017, "A computational method for automated detection of engineering structures with cyclic symmetries," Computers \& Structures, 191,153-164. 